\title{
Comparative transcriptome analysis of two selenium-accumulating genotypes of Aegilops tauschii Coss. in response to selenium
}

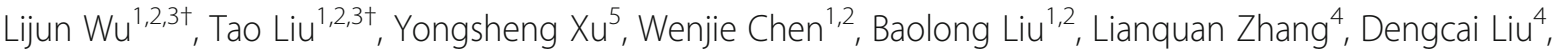 \\ Huaigang Zhang ${ }^{1,2,3^{*}}$ and Bo Zhang ${ }^{1,2^{*}}$ (D)
}

\begin{abstract}
Background: Selenium (Se), an essential micronutrient in both animals and humans, has various biological functions, and its deficiency can lead to various diseases. The most common method for increasing Se uptake is the consumption of Se-rich plants, which transform inorganic Se into organic forms. Wheat is eaten daily by many people. The Se content of Aegilops tauschii (Ae. tauschii), one of the ancestors of hexaploid common wheat, is generally higher than that of wheat. In this study, two genotypes of Ae. tauschii with contrasting Se-accumulating abilities were subjected to different Se treatments followed by high-throughput transcriptome sequencing.

Results: Sequencing of 12 transcriptome libraries of Ae. tauschii grown under different Se treatments produced about a total of 47.72 GB of clean reads. After filtering out rRNA sequences, approximately 19.3 million highquality clean reads were mapped to the reference genome (ta IWGSC_MIPSV2.1 genome DA). The total number of reference genome gene is 32,920 and about 26,407 known genes were detected in four groups. Functional annotation of these mapped genes revealed a large number of genes and some pivotal pathways that may participate in Se metabolism. The expressions of several genes potentially involved in Se metabolism were confirmed by quantitative real-time PCR.
\end{abstract}

Conclusions: Our study, the first to examine Se metabolism in Ae. tauschii, has provided a theoretical foundation for future elucidation of the mechanism of Se metabolism in this species.

Keywords: Differentially expressed genes, Selenium, Aegilops tauschii

\section{Background}

Selenium (Se) is an essential trace element with a variety of biological functions [1,2], and its deficiency can cause a series of health problems [3]. Se in wheat grains has high bioavailability [4]. Bread wheat (Triticum aestivum L., genome AABBDD), a major staple crop worldwide, is humanity's main food source. Bread wheat is an allohexaploid that originated from hybridization between cultivated tetraploid wheat $(T$.

\footnotetext{
* Correspondence: hgzhang@nwipb.cac.cn; zhangbo@nwipb.cas.cn ${ }^{\dagger} \mathrm{Li}$ Jun Wu and Tao Liu contributed equally to this work.

${ }^{1}$ Key Laboratory of Adaptation and Evolution of Plateau Biota (AEPB), Northwest Institute of Plateau Biology, Chinese Academy of Sciences, 23\# Xinning Lu, Xining 810008, Qinghai, China

Full list of author information is available at the end of the article
}

turgidum L., BBAA) and the diploid wheat relative Aegilops tauschii Coss. (DD). A previous study has indicated that Aegilops tauschii (Ae. tauschii) possesses a higher ability to accumulate Se than does bread wheat [5].

The final content of Se in plants is controlled by three processes: uptake of Se from soil, assimilation of Se, and translocation into different organs. An understanding of the molecular mechanisms of Se uptake, assimilation, and translocation would thus facilitate the development of cereal crops with increased Se grain contents [6]. One important tool to aid elucidation of these mechanisms is RNA-seq technology.

(c) The Author(s). 2019 Open Access This article is distributed under the terms of the Creative Commons Attribution 4.0 International License (http://creativecommons.org/licenses/by/4.0/), which permits unrestricted use, distribution, and reproduction in any medium, provided you give appropriate credit to the original author(s) and the source, provide a link to the Creative Commons license, and indicate if changes were made. The Creative Commons Public Domain Dedication waiver (http://creativecommons.org/publicdomain/zero/1.0/) applies to the data made available in this article, unless otherwise stated. 
In the present study, the transcriptome sequences of two Ae. tauschii accessions with different Se-accumulating abilities grown under various Se treatments were generated by RNA-seq, de novo assembled, and annotated. Comparative transcriptome analysis of leaves of the two genotypes was performed to reveal the differences in their transcriptional responses to Se treatment and to determine the genetic basis of their different Se accumulation capabilities. To improve understanding of Se metabolism in Ae. tauschii [7], bioinformatic tools were applied to investigate the pathways and genes expressed under $\mathrm{Se}$ treatment. The results of this study provide new insights into the molecular mechanisms underlying Se accumulation in Ae. tauschii, which should aid the development of new, more efficient methods to improve the Se contents of Ae. tauschii, or even wheat via molecular breeding.

\section{Results}

For use in this study, we selected Ae. tauschii ssp. strangulata AS2407 and Ae. tauschii ssp. tauschii AS65, which have the highest and lowest Se levels, respectively, of 10 different frequently used accessions [8]. To test the effect of Se on Ae. tauschii, pre-treated plants were cultivated hydroponically in plastic containers containing quarter-strength modified Hoagland's nutrient solution under a continuous oxygen supply.

\section{RNA sequencing of leaf transcriptomes of the two genotypes}

RNA-seq followed by strict quality control and processing generated a total of $47.72 \mathrm{~GB}$ of clean data from 12 transcriptome libraries. The 12 transcriptome libraries represented four treatment groups with three repetitions: CK1 (AS65 treated with water), CK2 (AS2407 treated with water), T1 (AS65 treated with $10 \mu \mathrm{M} \mathrm{Na} \mathrm{SeO}_{3}$ ), and T2 (AS2407 treated with $10 \mu \mathrm{M} \mathrm{Na}_{2} \mathrm{SeO}_{3}$ ). After filtering out duplicate sequences and ambiguous and low-quality reads, an average of $31.42 \times 10^{6}$ high-quality (HQ) clean reads remained per library: 32.14 million for CK1, 29.68 million for CK2, 31.98 million for T1, and 33.46 million for T2. Excluding rRNA sequences, this corresponded to 27.54 to 30.95 million High-quality clean reads (HQ clean reads) per group (Table 1; see Additional file 1: Data S2 for details). The average GC percentage was $57.83 \%$, with a QC30 base percentage above $95.45 \%$. Details on data and data quality before and after filtering are shown in Additional file 2: Figure S1 and Additional file 3: Data S1. All transcriptome data are shown in Table 1.

\section{Mapping of RNA sequences to the reference genome} HQ clean reads, except for rRNA, were mapped to the Ae. tauschii reference genome (ta IWGSC_MIPSv2.1 genome DA). Approximately 19.3 million clean reads (64.34\% of the total) were mapped; 0.33 million were multiply mapped, and 18.97 million were unique. An overview and detailed data are given in Table 2 and Additional file 4: Data S3.

\section{Gene expression statistics}

The total number of reference genome gene is 32,920 and about 26,407 known genes (about $80.22 \%$ ) were detected in four groups. New genes accounted for $19.66 \%$ of the total. The number of genes in each group and sample is summarized in Table 3 and Additional file 5: Data S4, respectively.

To check the reliability of the expression data, we compared the results among replicates. Pairwise Pearson correlation coefficients were calculated between replicates and represented as a heat map, with the intensity of the correlation indicated with a color scale. As can be seen on the correlation heat map of Additional file 6: Figure S2, the expression data of CK1-3 was not only very different from the other two replicates, but also the other groups. Because this aberrant expression indicates that the CK1-3 sample was contaminated or damaged, the CK1-3 data were not used in subsequent analyses.

A comparison of changes in gene expression between different groups revealed considerable differences. As a result of deep sequencing of the four groups, more than 27,000 genes $(82.02 \%)$ in the Ae. tauschii reference genome were detected in each group. The samples exhibited transcriptional complexity in terms of the different genotypes and Se treatments.

\section{Distribution of expressed genes}

Venn diagram analysis of Ae. tauschii genes coexpressed in the four groups revealed 26,123 coexpressed in CK1 and CK2, 26,776 in both CK1 and T1, 28,751 in both CK2 and T2, 27,356 in both T1 and T2. The number of specifically expressed genes in each group was as

Table 1 Sequencing and statistics for the four transcriptome data after Se treatment

\begin{tabular}{|c|c|c|c|c|}
\hline Group name & CK1 & CK2 & $\mathrm{T} 1$ & $\mathrm{~T} 2$ \\
\hline No. of Clean Reads $\left(\times 10^{6}\right)$ & $32.14 \pm 6.72$ & $29.68 \pm 4.38$ & $31.98 \pm 0.90$ & $33.46 \pm 2.94$ \\
\hline No. of HQ Clean Reads $\left(\times 10^{6}\right)$ & $31.75 \pm 6.67$ & $29.31 \pm 4.31$ & $31.58 \pm 0.91$ & $33.03 \pm 2.89$ \\
\hline rRNA Mapped Reads & $1.06 \pm 0.57$ & $1.76 \pm 0.56$ & $0.63 \pm 0.17$ & $2.12 \pm 1.06$ \\
\hline No. of $\mathrm{HQ}$ clean reads except the rRNA $\left(\times 10^{6}\right)$ & $30.69 \pm 6.27$ & $27.54 \pm 4.43$ & $30.95 \pm 0.99$ & $30.90 \pm 3.13$ \\
\hline
\end{tabular}

No. is short for number 
Table 2 Sequencing and statistics for the four group's transcriptome data with the reference genome (ta IWGSC_MIPSV2.1 genome DA)

\begin{tabular}{lllll}
\hline Group name & CK1 & CK2 & T1 & T2 \\
\hline No. of total reads $\left(\times 10^{6}\right)$ & $30.69 \pm 6.27$ & $27.54 \pm 4.43$ & $30.95 \pm 0.99$ & $30.90 \pm 3.13$ \\
No. of mapped reads $\left(\times 10^{6}\right)$ & $19.16 \pm 4.02$ & $18.24 \pm 3.00$ & $19.38 \pm 0.59$ & $20.42 \pm 1.90$ \\
Mapped percentage & $62.48 \% \pm 1.23 \%$ & $66.21 \% \pm 0.46 \%$ & $62.63 \% \pm 0.50 \%$ & $66.10 \% \pm 1.02 \%$ \\
Unique Mapped Reads $\left(\times 10^{6}\right)$ & $18.84 \pm 3.95$ & $17.94 \pm 2.95$ & $19.04 \pm 0.57$ & $20.06 \pm 1.86$ \\
Multiple Mapped reads $\left(\times 10^{6}\right)$ & $0.32 \pm 0.08$ & $0.30 \pm 0.05$ & $0.34 \pm 0.02$ & $0.36 \pm 0.05$ \\
\hline
\end{tabular}

follows: 192 (CK1), 411 (CK2), 590 (T1), and 581 (CK2) (Fig. 1; see Additional file 7: Data S5 for a list of genes by category). In addition, 25,315 genes were coexpressed in all four groups. Some genes, probably related to housekeeping functions, exhibited little variation across different groups, while others were quantitatively regulated. The dynamic nature of gene expression in the four samples suggests that the different genotypes and Se treatments affected the absorption of Se in Ae. tauschii.

To obtain statistical confirmation of differences in gene expression among the four groups, we normalized gene expression levels using the Fragments Per Kilobase of exon model per Million mapped reads (FPKM) method. The expressions of genes were classified according to their calculated FPKMs as low (0-10), medium (10-50), or high (>1000). The greatest proportion of genes in each group was those lowly expressed, with the smallest number expressed at a high level (Table 4). With or without Se treatment, more genes were expressed in AS2407 than AS65. Although the relationship trend between the number and percentage of expressed genes was consistent between genotypes, little difference was observed between Se and control treatments within a given genotype. In the present study, genotype had a more obvious effect on gene expression than exposure to $10 \mu \mathrm{M} \mathrm{Na}_{2} \mathrm{SeO}_{3}$.

\section{Analysis of differentially expressed genes (DEGs)}

To uncover significantly expressed genes between groups, genes differentially expressed between the two genotypes after $48 \mathrm{~h}$ under different Se conditions were identified using edgeR software with a false discovery rate (FDR)-adjusted $p$-value threshold [9, 10]. Transcripts with at least a two-fold difference in expression ( $\mid \log _{2}$ fold change $\mid>1$ ) and a FDR $<0.05$ were considered to be differentially expressed between groups [11].

Table 3 Detection gene number statistics for each group

\begin{tabular}{llll}
\hline Group name & Known gene num & New gene num & All gene num \\
\hline CK1 & $23,803(72.31 \%)$ & 5611 & 29,414 \\
CK2 & $24,271(73.73 \%)$ & 5719 & 29,990 \\
T1 & $23,515(71.43 \%)$ & 5589 & 29,104 \\
T2 & $24,311(73.85 \%)$ & 5745 & 30,056 \\
\hline
\end{tabular}

In the absence of Se, 5052 genes were differentially expressed between CK1 and CK2, among which 4533 were up-regulated and 1938 were down-regulated in CK2 compared with CK1 (CK1 vs. CK2). Under $10 \mu \mathrm{M}$ Se, we detected 4315 DEGs in T1 vs. T2, 2856 up-regulated and 1459 down-regulated. In AS65, 32 DEGs (27 up-regulated and 5 down-regulated) were expressed in CK1 vs. T1, while 322 DEGs (132 up-regulated and 140 down-regulated) were detected between CK2 and T2 in AS2407 (Fig. 2). These results suggest that the number of DEGs between genotypes were more than those between Se treatments. At $10 \mu \mathrm{M}$ $\mathrm{Na}_{2} \mathrm{SeO}_{3}$, the maximum concentration that can safely be applied to Ae. tauschii, the influence of added Se was much smaller than that of genotype.

An RNA-seq approach was used to preliminarily identify and analyze the differential expression of Ae. tauschii genes associated with Se. Because the two different genotypes pretreated with Se exhibited obvious changes in gene expression, DEGs between the four treatment groups were examined more extensively (Fig. 1). Among these DEGs, seven genes encoding the following proteins were regulated in an Se-related manner (Fig. 3): disease resistance protein RGA2-like (predicted, gene ID: XLOC_024936), Auxilin-related protein 1 (gene ID: XLOC_034135), protein transport protein SEC23-like (gene ID: XLOC_038938), Hessian fly response gene 1 protein (gene ID: XLOC_046021), Chalcone synthase 1 (gene ID: Traes_2DL_BC7F606B9), Fatty acyl-CoA reductase 1 (gene ID: Traes_5DL_5597A11EC) and beta-amylase (gene ID: Traes_2DS_D14ABD1DB). The seven proteins known to interact with Se were verified by real-time fluorescence quantitative PCR (RT-qPCR) (Additional file 8: Figure S3). There is a certain error that the fold changes were very high because the FPKM of one simple was almost zero and another simple was less than 10 in the RNA-seq data sequencing. This happened in gene XLOC_024936 and XLOC_034135. As indicated by the general correlation coefficient $\left(R^{2}=0.9275\right)$ (exclusive of gene XLOC_024936 and XLOC_034135), a good concordance was observed between the expression patterns of DEGs obtained by RNA-seq and RT-qPCR data. Chalcone synthase 1 is involved in flavonoid and secondary metabolite biosynthetic pathways. Genes regulating secondary metabolite biosynthesis are interesting 


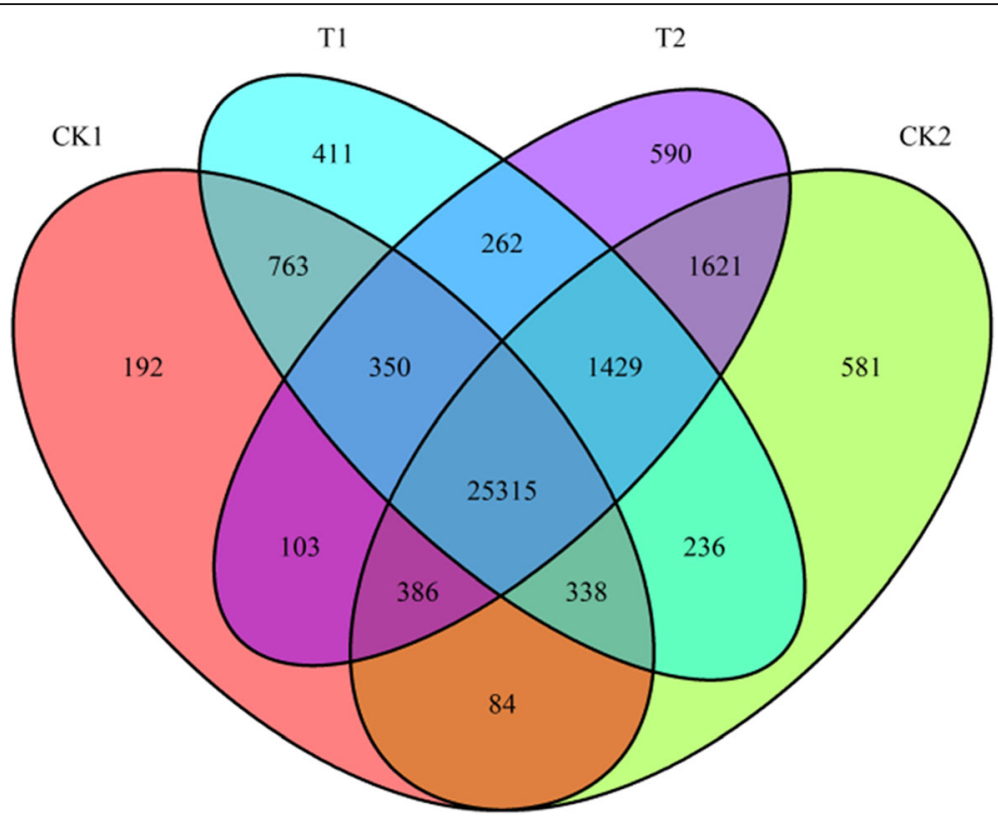

Fig. 1 The Venn diagram is exhibiting the overlaps in four groups

candidates for further physiological and molecular investigations of Se stress tolerance in Ae. tauschii.

\section{Functional analysis of Se-influenced genes}

To facilitate global analysis of gene expression, we investigated the possible mechanisms of significant DEGs by Gene Ontology (GO) analysis (Fig. 4). The $13,354,99,635$, and 9775 significant (q-value <0.05) DEGs in CK1 vs. CK2, CK1 vs. T1, CK2 vs. T2, and T1 vs. T2, respectively, were categorized into $42,24,23$, and $42 \mathrm{GO}$ terms in the three main GO categories of biological process, cellular component, and molecular function (Fig. 4). DEG- enriched GO terms in CK1 vs. CK2 and T1 vs. T2 were identical. The mostly highly enriched GO terms in CK1 vs. CK2, CK1 vs. T1, CK2 vs. T2, and T1 vs. T2 were catalytic activity (GO: 0003824; 2014, 14, 129, and 1382 genes, respectively, binding (GO: 0005488; 2144, 10, 97, and 1556 genes), cellular process (GO: 0009987; 1520, 8, 67, and 1141 genes), and metabolic process (GO: 0008152; 2227, 16, 134, and 1512 genes). The genes in these different expression clusters associated with different GO terms clearly indicate biological processes and molecular functions that are Se-related.

Kyoto Encyclopedia of Genes and Genomes (KEGG) pathway analysis was used to assign 1136 DEGs in CK1 vs. CK2 to 125 pathways, 14 in CK1 vs. T1 to 22 pathways, 60 in CK2 vs. T2 to 64 pathways, and 740 in T1 vs. T2 to 121 pathways. The most obviously enriched metabolic pathways $(q$-value $\leq 0.05)$ in CK1 vs. CK2, CK2 vs. T2, and T1 vs. T2 respectively totaled 16,8 , and 6. Cutin, suberin and wax biosynthesis (ko00073) and flavonoid biosynthesis (ko00941) were the most common obviously enriched metabolic pathways in CK1 vs. CK2 and CK2 vs. T2, while the following pathways were especially enriched in CK2 vs. T2: anthocyanin biosynthesis (ko00942), beta-alanine metabolism (ko00410), pyruvate metabolism (ko00620), tyrosine metabolism (ko00350), isoquinoline alkaloid biosynthesis (ko00950), and ascorbate and aldarate metabolism (ko00053). In CK1 vs. CK2 and T1 vs. T2, the most common highly enriched metabolic pathways were plant-pathogen interaction (ko04626), glutathione metabolism (ko00480), fructose and mannose metabolism

Table 4 Statistics of genes in different expression-level interval

\begin{tabular}{lllll}
\hline FPKM interval & CK1 (\%) & CK2 (\%) & T1 (\%) & T2 (\%) \\
\hline $0-10$ & $16,836(61.15)$ & $18,187(60.64)$ & $18,326(62.97)$ & $18,486(61.51)$ \\
$10-50$ & $7600(19.88)$ & $8414(20.13)$ & $7791(19.53)$ & $8184(19.66)$ \\
$50-100$ & $1635(5.34)$ & $1770(5.30)$ & $1565(4.88)$ & $1735(5.19)$ \\
$100-500$ & $1220(4.21)$ & $1381(4.37)$ & $1194(3.91)$ & $1407(4.44)$ \\
$500-1000$ & $125(0.45)$ & $139(0.46)$ & $117(0.4)$ & $139(0.46)$ \\
$>1000$ & $115(0.42)$ & $99(0.33)$ & $112(0.38)$ & $105(0.35)$ \\
\hline
\end{tabular}




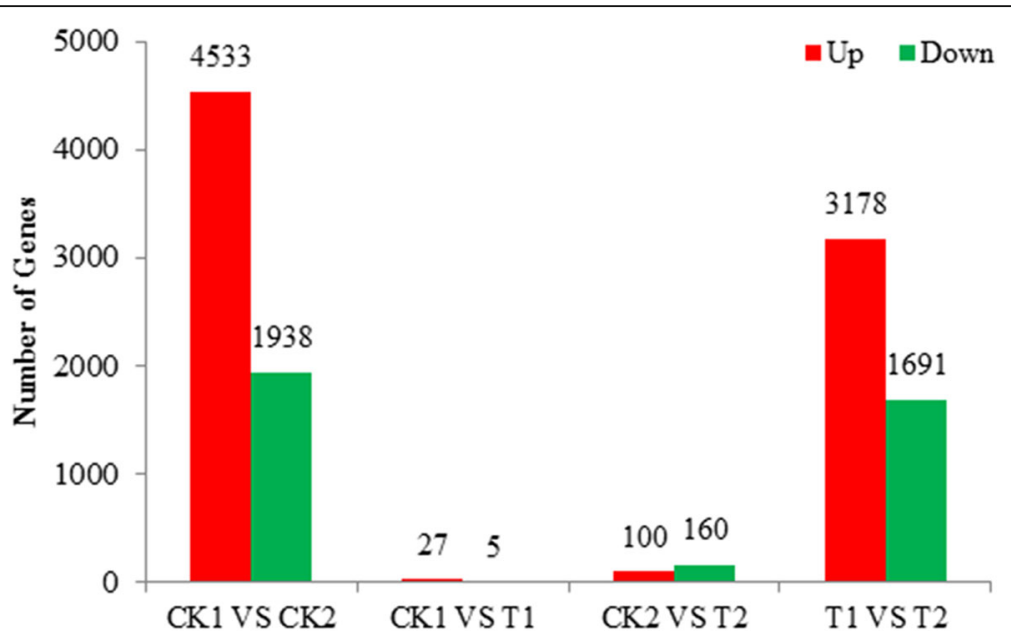

Fig. 2 Changes in gene expression profile between different groups

(ko00051), phenylpropanoid biosynthesis (ko00940), and aminoacyl-tRNA biosynthesis (ko00970). Phenylalanine metabolism (ko00360) was the only obviously enriched metabolic pathway in T1 vs. T2. Although flavonoid biosynthesis was an enriched metabolic pathway in each comparison group, its $q$-value was greater than 0.05 in CK1 vs. T1 and T1 vs. T2.

For elucidating the useful information related to selenium accumulation, expressed genes in each group of CK1 vs. T1 and CK 2 vs. T2 were compared. The 83 and 619 unique DEGs in CK1 vs. T1 and CK2 vs. T2 were respectively categorized into 24 and 24 GO terms in the three main GO categories of biological process, cellular component, and molecular function (Additional file 9: Data S6). Interestingly, the unique DEGs in the two groups were categorized into the same $21 \mathrm{GO}$ terms related to cellular process, metabolic process, membrane, catalytic activity, transporter activity, and so on. The 10 and 65 unique DEGs in each CK1 vs. T1 and CK2 vs. T2 were respectively categorized to 17 and 63 pathways, among them 10 pathways were common (Additional file 10: Data S7). ABC transporters, glutathione metabolism, Phenylalanine metabolism, Circadian rhythm - plant, Flavonoid biosynthesis, and

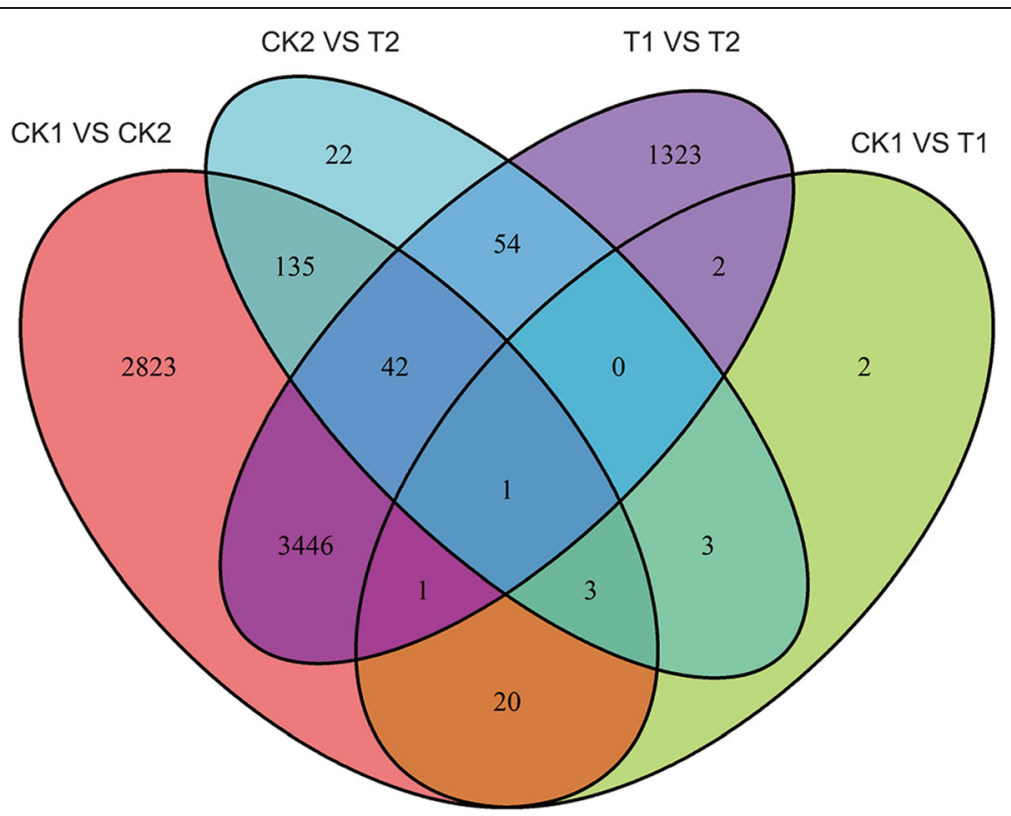

Fig. 3 The Venn diagram shows the differentially expressed genes between each two groups and the number of overlapping expression of genes 


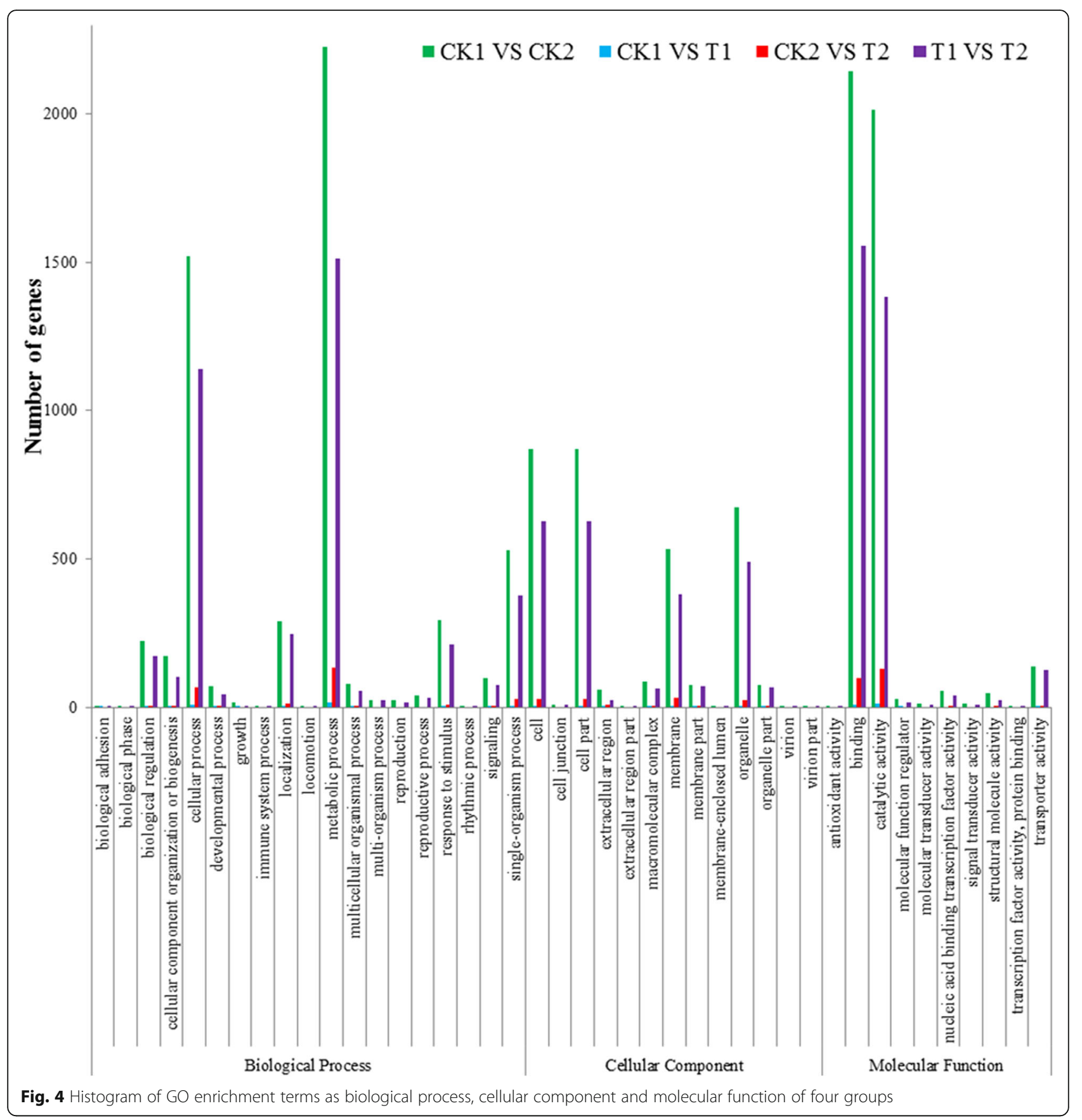

Glycolysis / Gluconeogenesis were found in unique CK2 vs. T2.

\section{Experimental validation of DEGs by RT-qPCR}

To evaluate the validity of the RNA-seq data, we carried out a RT-qPCR analysis of eight DEGs involved in flavone and flavonol biosynthesis [7], flavonoid biosynthesis [7], and selenocompound metabolism which were believed to be related to selenium metabolism. As shown in Fig. 5, the expression patterns determined by RT-qPCR were in agreement with those based on
RNA-seq data. As indicated by the general correlation coefficient $\left(R^{2}=0.8433\right)$, a good concordance was observed overall between the expression patterns of DEGs obtained by RNA-seq and RT-qPCR data, thus supporting the reliability of the data.

\section{Transcription factors (TFs) related to Se}

Many putative TF genes, including bHLH, WRKY, MYB, and AP2 (Table 5 and Additional file 11: Data S8), were identified by querying the Plant Transcription Factor Database (http://planttfdb.cbi.pku.edu.cn/). The expression 


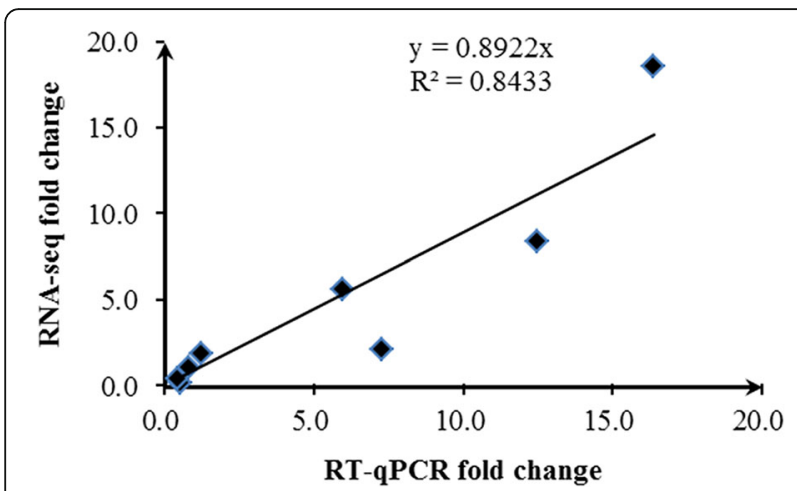

Fig. 5 Correlation of fold change values between RNA-seq and RT-qPCR. The $R^{2}$ value is 0.8433

trends of differentially expressed TFs in CK1 vs. CK2 were consistent with those common to T1 vs. T2; the same was true between CK1 vs. T1 and CK2 vs. T2. Most of the common TFs were down-regulated. M-type MADS genes were all down-regulated in CK1 vs. T1 and CK2 vs. T2-in other words, after Se treatment. Genes encoding AP2 proteins were down-regulated in CK1 vs. CK2 and T1 vs. T2, while a gene (Traes_5DL_32D78D06A) encoding the WRKY TF was up-regulated in all groups.

Except for common TFs, TFs in each comparison group (Additional file 11: Data S8), including NAC, WRKY. and bHLH, were mostly up-regulated. MYB TFs in CK1 vs. T1 and in CK2 vs. T2 were down- regulated, whereas bHLH TFs in CK1 vs. CK2 and in T1 vs. T2 were all up-regulated.

\section{Discussion}

Selenium is an essential trace mineral of fundamental importance to human health [12]. Humans rely on plants, including cereal crops, as a medium to supplement nutritional Se. The Se hyperaccumulator Astragalus bisulcatus and the secondary Se accumulator Brassica oleracea var. italica (broccoli) are well-known models for investigating the molecular and biochemical basis of Se in plants [13]. In contrast, studies related to Se metabolism in Triticeae crops have not been reported.

The advent of RNA-seq has simplified the acquisition of transcriptome data from plant tissues or cells under specific conditions [7]. In the present study, we thus aimed to elucidate the molecular basis of Se metabolism in Ae. tauschii by RNA-seq. A total of $47.72 \mathrm{~GB}$ of clean data were generated, with 32.14, 29.68, 31.98, and 33.46 million clean reads respectively obtained from CK1, CK2, T1, and T2 samples after filtering out low-quality reads. Approximately 19.3 million clean reads $(64.34 \%$ of the total) were mapped to the $A e$. tauschii reference genome (ta IWGSC_MIPSv2.1 genome DA). A total of 26,407 known genes were detected in the four treatment groups, with unidentified genes accounting for $19.66 \%$ of genes inferred from the reference genome. We uncovered 25,315 genes coexpressed in all four groups, while some genes were coexpressed in two or three groups (Fig. 1). Statistical confirmation of gene expression differences among the four groups demonstrated that genotype was a more obvious influence than treatment with $10 \mu \mathrm{M} \mathrm{Na} \mathrm{NeO}_{3}$. Analysis of DEGs in the four groups further strengthened the observation that the effect of Se concentration was much smaller than that of genotype. The relatively inconsequential effect of added $\mathrm{Na}_{2} \mathrm{SeO}_{3}$ may be mainly because a concentration of $10 \mu \mathrm{M}$ was insufficient to

Table 5 Common difference expression transcription factors in four groups

\begin{tabular}{|c|c|c|c|c|c|c|c|c|c|c|}
\hline \multirow[t]{2}{*}{ Group } & \multirow[t]{2}{*}{ Gene ID } & \multirow[t]{2}{*}{ Description } & \multirow{2}{*}{$\begin{array}{l}\text { CK1 } \\
\text { FPKM }\end{array}$} & \multirow{2}{*}{$\begin{array}{l}\text { CK2 } \\
\text { FPKM }\end{array}$} & \multirow{2}{*}{$\begin{array}{l}\text { T1 } \\
\text { FPKM }\end{array}$} & \multirow{2}{*}{$\begin{array}{l}\text { T2 } \\
\text { FPKM }\end{array}$} & \multicolumn{4}{|c|}{ Fold change } \\
\hline & & & & & & & $\begin{array}{l}\text { CK1 VS } \\
\text { CK2 }\end{array}$ & $\begin{array}{l}\text { CK1 VS } \\
\text { T1 }\end{array}$ & $\begin{array}{l}\text { CK2 VS } \\
\mathrm{T} 2\end{array}$ & $\begin{array}{l}\text { T1 VS } \\
\text { T2 }\end{array}$ \\
\hline \multirow[t]{3}{*}{$\overline{\mathrm{bHLH}}$} & XLOC_013854 & Transcription factor PIF3 & 12.33 & 42.66 & 15.73 & 73.21 & 3.46 & 1.28 & 1.72 & 4.65 \\
\hline & Traes_2DL_865346DA2 & Transcription factor ORG2 & 73.52 & 19.92 & 67.07 & 17.30 & 0.27 & 0.91 & 0.87 & 0.26 \\
\hline & XLOC_014379 & Transcription factor bHLH93 & 14.73 & 3.90 & 15.19 & 3.31 & 0.26 & 1.03 & 0.85 & 0.22 \\
\hline WRKY & Traes_5DL_32D78D06A & WRKY transcription factor & 2.70 & 77.10 & 5.04 & 112.02 & 28.56 & 1.87 & 1.45 & 22.23 \\
\hline \multirow[t]{2}{*}{ MYB } & Traes_7DL_090D0E08E & Myb family transcription factor APL & 15.45 & 33.52 & 15.41 & 36.98 & 2.17 & 1.00 & 1.10 & 2.40 \\
\hline & XLOC_006616 & Myb family transcription factor APL & 44.25 & 5.61 & 30.46 & 4.96 & 0.13 & 0.69 & 0.88 & 0.16 \\
\hline \multirow{3}{*}{$\begin{array}{l}\text { M-type } \\
\text { MADS }\end{array}$} & Traes_3DL_915546ED4 & MADS-box transcription factor TaAGL41 & 25.23 & 59.11 & 23.01 & 48.34 & 2.34 & 0.91 & 0.82 & 2.10 \\
\hline & Traes_4DL_C4CB3D5AF & MADS-box transcription factor TaAGL33 & 27.75 & 62.73 & 26.11 & 54.00 & 2.26 & 0.94 & 0.86 & 2.07 \\
\hline & Traes_3DL_FBB80151F & MADS-box transcription factor TaAGL42 & 26.65 & 1.51 & 26.76 & 1.42 & 0.06 & 1.00 & 0.94 & 0.05 \\
\hline \multirow[t]{3}{*}{ AP2 } & XLOC_004436 & $\begin{array}{l}\text { AP2-like ethylene-responsive transcription } \\
\text { factor ANT }\end{array}$ & 15.32 & 0.42 & 19.20 & 0.85 & 0.03 & 1.25 & 2.02 & 0.04 \\
\hline & XLOC_028083 & $\begin{array}{l}\text { AP2-like ethylene-responsive transcription } \\
\text { factor ANT }\end{array}$ & 41.35 & 2.82 & 50.86 & 3.36 & 0.07 & 1.23 & 1.19 & 0.07 \\
\hline & XLOC_040922 & AP2/EREBP type transcription factor & 43.00 & 3.70 & 28.89 & 5.81 & 0.09 & 0.67 & 1.57 & 0.20 \\
\hline
\end{tabular}


counterbalance the differences between genotypes. Co-DEGs in CK1 vs. T1 and CK2 vs. T2 were verified and a good concordance $\left(R^{2}=0.9275\right)$ was observed between the expression patterns of DEGs obtained by RNA-seq and RT-qPCR data, thus supporting the reliability of the data. Genes regulating secondary metabolite biosynthesis, such as chalcone synthase 1 preliminarily identified by analysis of Se-associated DEGs, are interesting candidates for further physiological and molecular investigations of Se stress tolerance in Ae. tauschii. The five co-DEGs (protein transport protein SEC23-like, Hessian fly response gene 1 protein, Chalcone synthase 1, Fatty acyl-CoA reductase 1 and beta-amylase) will be the potential candidates for Se related function analysis in our future study. GO enrichment analysis of significant DEGs revealed the biological processes and molecular functions involved in Se absorption. Flavonoid biosynthesis was the most common obviously enriched metabolic pathway in AS2407 vs. AS65 (both treated with water) and Se-treated vs. water-treated AS2407. Metabolites possibly useful for plant adaptation to the environment are thought to be produced via flavone and flavonol biosynthesis, flavonoid biosynthesis pathways [7]. In addition, phenylalanine metabolism (ko00360) was an especially highly enriched metabolic pathway in Se-treated AS2407 compared with Se-treated AS65. DEGs associated with flavonoid biosynthesis and phenylalanine metabolism should be further explored in future research related to Se. ABC transporters, Phenylalanine metabolism, Circadian rhythm - plant, Flavonoid biosynthesis, and Glycolysis / Gluconeogenesis enriched in unique CK2 vs. T2 compared with CK1 vs. T1 which were consistent with Özgür Çakır [7] were be regarded as related to the metabolism of selenium. Selenium is an important component of glutathione peroxidase in glutathione metabolism which was also found in unique CK2 vs. T2 compared with CK1 vs. T1. Se treatment made the above pathways responded in AS2407 (T2). The unique DEGs in CK2 vs. T2 may be the reason why the content of Se in AS2407 was higher than in AS65.

Eight DEGs involved in flavone and flavonol biosynthesis, flavonoid biosynthesis, and selenocompound metabolism was performed by RT-qPCR to evaluate the validity of the RNA-seq data, the result was in agreement. But a small amount of difference was uncovered, and it may be caused by differences in the algorithms used to determine expression levels [14].

All major life processes depend on differential gene expression, a phenomenon largely controlled by TFs [15]. Many TF families may play important roles in developmental processes [16] in which TFs can act as activators, repressors, or both [15]. Se treatment may cause aberrant expression of many protein-coding genes [7]. M-type MADS proteins are involved the control of flower development in plants [17], while AP2 encoding proteins participate in the regulation of processes such as flower and embryo development [18]. WRKY proteins are a superfamily of TFs [19] that play important roles in responses to abiotic stress [20-22]. These TFs are related to plant development and respond to a variety of stresses [23]. In our study, WRKY genes were mostly up-regulated under Se treatment, a result in agreement with the finding that selenite treatment up-regulates genes encoding the WRKY TF family in Astragalus chrysochlorus [24]. MYB genes in CK1 vs. $\mathrm{T} 1$ and CK2 vs. T2 were down-regulated in our study; this is contrary to the results of Agarwal et al. [25], but different concentrations and chemical forms of Se were used in their study. The bHLH TFs can regulate flavonoid, alkaloid, and terpene biosynthesis [26, 27], which is consistent with our results. Flavonoid biosynthesis, which is somewhat related to environmental adaptation and stress response, is affected by Se treatment [7]. bHLH TF genes differentially expressed between the two genotypes in our study are likely associated with Se treatment.

\section{Conclusions}

In this study, we used gene expression data to analyze differences in transcription-level responses of two genotypes of Ae. tauschii to Se. The number of DEGs in Se- vs. water-treated AS2407 was larger than in similarly treated AS65 plants, a finding consistent with the greater Se-absorbing ability of AS2407 vs. AS65 [8]. The five co-expression genes (protein transport protein SEC23-like, Hessian fly response gene 1 protein, Chalcone synthase 1 , Fatty acyl-CoA reductase 1 and beta-amylase) in CK1 vs. $\mathrm{T} 1$ and CK2 vs. T2 and eight DEGs involved in flavone and flavonol biosynthesis, flavonoid biosynthesis, and selenocompound metabolism were believed to be potentially related to selenium metabolism. The pathways (ABC transporters, Phenylalanine metabolism, Circadian rhythm plant, Flavonoid biosynthesis, and Glycolysis / Gluconeogenesis and glutathione metabolism) enriched by unique DEGs in CK2 vs. T2 compared with CK1 vs. T1 were regarded as related to the metabolism of selenium and it may be the reason why the content of Se in AS2407 was higher than in AS65. Finally, our results may help inform the interpretation of some TFs and metabolic modifications related to Se as all of these reactions may occur between the modifications in gene expression and the resulting physiological response.

\section{Methods}

\section{Plant materials and Se treatments}

Ae. tauschii. ssp. strangulata AS2407 and Ae. tauschii ssp. tauschii AS65 were selected for use in this study. Seeds of both accessions were obtained from Sichuan Agricultural University, Sichuan Province, China. The seeds were soaked in deionized water at room temperature for $24 \mathrm{~h}$ 
and then germinated on moist filter paper in Petri dishes at $24 \pm 1{ }^{\circ} \mathrm{C}$ under a $16 / 8$-h light/dark cycle. Two-weekold germinated seedlings were transplanted from the Petri dishes to plastic containers containing quarter-strength modified Hoagland's nutrient solution in a greenhouse under the same temperature and photoperiod conditions [28]. The composition of the nutrient solution was as follows: $1.0 \mathrm{mM} \mathrm{Ca}\left(\mathrm{NO}_{3}\right)_{2} \cdot 4 \mathrm{H}_{2} \mathrm{O}, 1.25 \mathrm{mM} \mathrm{KNO} 3,0.25 \mathrm{mM}$ $\mathrm{NH}_{4} \mathrm{NO}_{3}, 0.25 \mathrm{mM} \mathrm{KH_{2 }} \mathrm{PO}_{4}, 0.95 \mathrm{mM} \mathrm{MgSO}, 1.25 \mu \mathrm{M}$ $\mathrm{KI}, 36.75 \mu \mathrm{M} \mathrm{MnSO}_{4}, 25 \mu \mathrm{MH}_{3} \mathrm{BO}_{3}, 13.35 \mu \mathrm{M} \mathrm{ZnSO}{ }_{4}$, $0.25 \mu \mathrm{M} \quad \mathrm{Na}_{2} \mathrm{MoO}_{4} \cdot 2 \mathrm{H}_{2} \mathrm{O}, 0.0475 \mu \mathrm{M} \quad \mathrm{CuSO} 4,0.05 \mu \mathrm{M}$ $\mathrm{CoCl}_{2}$, and $12.5 \mu \mathrm{M}$ Fe(III)-EDTA. After 40 days of growth, the plants were treated with fresh medium supplemented with $\mathrm{Na}_{2} \mathrm{SeO}_{3}$ to a final concentration of $10 \mu \mathrm{M}$, a level of Se that causes mild stress in Ae. tauschii but no observable toxic symptoms. After $72 \mathrm{~h}$, young leaf samples from at least three similarly sized Se-treated or control plants of each genotype were harvested separately. Fresh leaf tissues were frozen in liquid nitrogen and stored at $-80^{\circ} \mathrm{C}$ for RNA extraction.

\section{RNA extraction and RNA-Seq library construction and sequencing}

Total RNA was separately extracted from frozen leaf tissues of three samples per treatment using a Trizol kit (Promega, USA) following the manufacturer's protocol. The extracted RNA was treated with RNase-free DNase I (Takara Bio, Japan) for $30 \mathrm{~min}$ at $37^{\circ} \mathrm{C}$ to remove residual DNA. The quality of the purified RNA was verified by agarose gel electrophoresis, and its concentration was measured on a 2100 Bioanalyzer (Agilent Technologies, Santa Clara, CA, USA) at $260 \mathrm{~nm}$ and $280 \mathrm{~nm}$. High-quality RNA from the three sample replicates was combined for subsequent RNA sequencing.

The extracted RNA samples were used for cDNA synthesis. Poly (A) mRNA was isolated using oligo-dT beads (Qiagen) and broken into short fragments (200 nt) with fragmentation buffer. First-strand cDNA was generated by random hexamer-primed reverse transcription using the fragmented mRNA as a template, with second-strand CDNA then synthesized after addition of buffer, dNTPs, RNase $\mathrm{H}$, and DNA polymerase I. The generated cDNA fragments were purified using a QIAquick PCR gel extraction kit (Qiagen, Germany), washed with EB, subjected to end reparation and poly (A) addition, and ligated to sequencing adapters. cDNA fragments of interest $(200 \pm 25 \mathrm{bp})$ were purified and enriched by PCR amplification to complete preparation of the entire cDNA library. The constructed cDNA library was sequenced in a single run on the IlluminaHiSeq 2500 platform using single-end and paired-end technology at Beijing Genomics Institute (Shenzhen, China).
Clean reads were selected using a custom Perl script that removed low-quality sequences (more than $50 \%$ of bases with quality scores $<20$ ), reads with more than $5 \%$ unknown ("N") bases, and reads containing adaptor sequences. After removal of rRNA sequences from the clean reads, the remaining transcripts were aligned to the Ae. tauschii reference genome and used to reconstruct the transcriptome. RNA-seq data analysis was performed using several packages in the JAVA-based client-server system Chipster [29].

\section{Annotation and normalization of gene expression levels}

Gene abundances were quantified with RSEM software [30] and sequencing reads were mapped to the reference sequence ( $t a$ IWGSC_MIPSv2.1 genome D). First, a set of reference transcript sequences was generated and preprocessed. Second, RNA-seq reads were realigned to the reference transcripts using the Bowtie alignment program, and the resulting alignments were used to estimate gene abundances. Expression levels of all detected genes were obtained by calculating the expression level of this gene with the coverage of reads in one gene.

Gene expression levels were then normalized using the FPKM method according to the following formula:

$$
\operatorname{FPKM}(A)=\frac{10^{6} \mathrm{C}}{N L / 10^{3}}
$$

where FPKM $(A)$ is the expression of gene $A, C$ is the number of fragments mapped to gene $A, N$ is the total number of fragments mapped to reference genes, and $L$ is the number of bases on gene $A$ [31]. The FPKM value can be directly used to compare differences in gene expression among samples, as this method is able to eliminate the influence of different gene lengths and sequencing data amounts on gene expression calculations.

\section{Analysis of expression patterns}

Pairwise Pearson correlation coefficients were calculated between samples based on the expression of all gene sets. Correlations between each pair of samples were then visualized in the form of a correlation heat map using a function in R (http://www.r-project.org/).

After annotation of gene expression levels, identical genes were compared between samples. The FDR was used to determine the $p$-value threshold in multiple tests, with the criteria of FDR $<0.05$ and $\mid \log _{2}$ ratio $\mid>1$ used in subsequent analyses to judge the significance of gene expression differences. In the case of more than two samples, the union (or intersection) of all DEGs was used in subsequent analyses. When multiple transcripts mapped to the same gene, the longest transcript was used to calculate the gene expression level and coverage. 
GO and KEGG functional annotation and classification GO analysis, used to identify the main biological functions of DEGs, is an international standardized gene functional classification system that has three ontologies: molecular function, cellular component, and biological process. To determine their main biological functions, DEGs were annotated with terms in the GO database (http://www.geneontology.org/) using Blast2GO [32] according to their numeric orders in the nr database to determine the main biological functions. Genes usually interact with each other to play roles in certain biological functions. Pathway-based analysis helps to further understand gene biological functions. KEGG is the major public pathway-related database [33]. Pathway enrichment analysis was used to identify metabolic or signal transduction pathways significantly enriched in DEGs compared with the whole genome background. The calculating formula was the same as that used in the GO analysis as the calculated $p$-value adjusted based on a FDR $<0.05$ threshold. Pathways meeting this condition were defined as significantly enriched in DEGs.

\section{Excavation and functional annotation of new genes}

Novel genes and transcripts were explored and existing genome annotation information was complemented by comparing the original genome annotation information with the reference genome sequences. New genes were discovered using the annotation library NR [34] as well as KEGG sequence alignment [35] using BLAST [36] software. New gene annotation information was obtained from the homologous gene annotation information in the library.

\section{RT-qPCR}

Total RNA $(2 \mu \mathrm{g})$ from each sample was reverse transcribed into cDNA using a RevertAid First Strand cDNA Synthesis kit (Thermo Scientific) according to the manufacturer's instructions, and stored at $-20^{\circ} \mathrm{C}$. A total of 8 selected genes related to selenium were analyzed by RT-qPCR using the SYBR Green qPCR Master Mix (TaKaRa) and the ABI ViiA 7 real-time PCR system (Applied Biosystems). The primer sequences are listed in Additional file 12: Data S9. Relative quantification was calculated by the comparative $2^{-\Delta \Delta C t}$ method [37]. All reactions were performed in triplicates per sample.

\section{Additional files}

Additional file 1: Data S2. Information statistics of reads filtering and $\mathrm{HQ}$ clean data compared with rRNA. (XLS 26 kb)

Additional file 2: Figure $\mathbf{S}$ 1. Classification of raw reads in each sample. (TIF 809 kb)

Additional file 3: Data S1. Information statistics of data before and after filtering. (XLS $27 \mathrm{~kb}$ )
Additional file 4: Data S3. Comparison statistics of reads which were unmapped with rRNA compared with the reference genome. (XLS $25 \mathrm{~kb}$ )

Additional file 5: Data S4. General statistics of detected gene's number in each sample. (XLS $24 \mathrm{~kb}$ )

Additional file 6: Figure S2. Correlation heat maps of each sample. (TIF $459 \mathrm{~kb}$ )

Additional file 7: Data S5. Detailed genes list for each category. (XLS $1856 \mathrm{~kb}$ )

Additional file 8: Figure S3. Seven genes regulated in a Se-related were verified by RT-qPCR. (TIF $204 \mathrm{~kb}$ )

Additional file 9: Data S6. GO functional classification of unique DEGs in each group of CK1 vs.T1 and CK2 vs.T2. (XLSX 14 kb)

Additional file 10: Data S7. KEGG pathway annotation of unique DEGs in each group of CK1 vs.T1 and CK2 vs.T2. (XLSX $16 \mathrm{~kb}$ )

Additional file 11: Data S8. Characteristic transcription factor genes list between comparison groups. (XLS $46 \mathrm{~kb}$ )

Additional file 12: Data S9. List of primers used in RT-qPCR. (XLS 26 kb)

Abbreviations

DEGs: Differentially expressed genes; FDR: False discovery rate; FPKM: Fragments Per Kilobase of exon model per Million mapped reads; GO: Gene Ontology; HQ clean reads: High-quality clean reads; KEGG: Kyoto Encyclopedia of Genes and Genomes; RT-qPCR: Real-time fluorescence quantitative PCR; TFs: Transcription factors

\section{Acknowledgements}

The authors thank the Gene Denovo Co. (Guangzhou, China) for the assistance with data processing and bioinformatics analysis.

\section{Fundings}

Lijun Wu is supported by the STS Project of Chinese Academy of Sciences (KFJ-STS-ZDTP-024CAS), Natural Science Foundation of China (No.31101140) and "Light of West China" Program. This research is funded in part by the Project from Qinghai Province (2016-HZ-808), the Strategic Priority Research Program of Chinese Academy of Sciences (No. XDA08030106) and the Program of Science and Technology Innovation Platform of Qinghai Province (2017ZJ-Y14). The funders played no role in study design, data collection and analysis, decision to publish, or preparation of the manuscript.

\section{Availability of data and materials}

Raw data supporting our findings can be found in the National Center for Biotechnology Information (NCBI) database under the accession number PRJNA505880.

Authors' contributions

LJW, TL, and BZ designed and conceived the experiment. YSX and LJW collected and processed the samples. LJW, TL, WJC, BLL, and LQZ performed the data analysis and draft the manuscript. BZ, DCL, and $H G Z$ helped to improve the manuscript. All authors read and approved the final manuscript.

Ethics approval and consent to participate

Our research did not involve any human or animal subjects, material, or data. All the plant materials used in this study were provided by Triticeae Research Institute, Sichuan Agricultural University, China. They are freely available for research purposes following institutional, national and international guidelines. The experiments were conducted under local legislation and permissions.

Consent for publication

Not applicable.

Competing interests

The authors declare that they have no competing interests.

\section{Publisher's Note}

Springer Nature remains neutral with regard to jurisdictional claims in published maps and institutional affiliations. 


\section{Author details}

Key Laboratory of Adaptation and Evolution of Plateau Biota (AEPB), Northwest Institute of Plateau Biology, Chinese Academy of Sciences, 23\# Xinning Lu, Xining 810008, Qinghai, China. ${ }^{2}$ Qinghai Provincial Key Laboratory of Crop Molecular Breeding, Xining 810008, China. ${ }^{3}$ University of Chinese Academy of Sciences, Beijing 100049, China. ${ }^{4}$ Triticeae Research Institute, Sichuan Agricultural University, Chengdu 611130, China. ${ }^{5}$ Xining Administration Center of Parks, Xining 810001, China

Received: 23 April 2018 Accepted: 26 November 2018

Published online: 14 January 2019

\section{References}

1. Zhu YG, Pilon-Smits EA, Zhao FJ, Williams PN, Meharg AA. Selenium in higher plants: understanding mechanisms for biofortification and phytoremediation. Trends Plant Sci. 2009;14(8):436-42.

2. Clark LC, Combs GF Jr, Turnbull BW, Slate EH, Chalker DK, Chow J, Davis LS, Glover RA, Graham GF, Gross EG, et al. Effects of selenium supplementation for cancer prevention in patients with carcinoma of the skin. A randomized controlled trial. Nutritional Prevention of Cancer Study Group. JAMA. 1996; 276(24):1957-63.

3. Lyons G, Stangoulis J, Graham R. High-selenium wheat: biofortification for better health. Nutr Res Rev. 2003;16(1):45-60.

4. Thomson CD. Assessment of requirements for selenium and adequacy of selenium status: a review. Eur J Clin Nutr. 2004;58(3):391-402.

5. Lyons G, Ortiz-Monasterio I, Stangoulis J, Graham R. Selenium concentration in wheat grain: is there sufficient genotypic variation to use in breeding? Plant Soil. 2005:269(1-2):369-80.

6. Zhao DY, Sun FL, Zhang B, Zhang ZQ, Yin LQ. Systematic comparisons of orthologous selenocysteine methyltransferase and homocysteine methyltransferase genes from seven monocots species. Notulae Scientia Biologicae. 2015;7(2):210-6.

7. Çakır Ö, Turgut-Kara N, Ari S, Zhang B. De novo transcriptome assembly and comparative analysis elucidate complicated mechanism regulating Astragalus chrysochlorus response to selenium stimuli. PLoS One. 2015; 10(10):e0135677.

8. Zhao DY, Zhang B, Chen WJ, Liu BL, Zhang LQ, Zhang HG, Liu DC Comparison of zinc, iron, and selenium accumulation between synthetic hexaploid wheat and its tetraploid and diploid parents. Can J Plant Sci. 2017;97:692-701.

9. Salkind N, editor. Encyclopedia of measurement and statistics. Thousand Oaks: Sage; 2007.

10. Rajkumar AP, Qvist P, Lazarus R, Lescai F, Ju J, Nyegaard M, Mors O, Borglum $A D, L i Q$, Christensen JH. Experimental validation of methods for differential gene expression analysis and sample pooling in RNA-seq. BMC Genomics. 2015;16:548.

11. Audic S, Claverie JM. The significance of digital gene expression profiles. Genome Res. 1997;7(10):986-95.

12. Rayman MP. The importance of selenium to human health. Lancet. 2000; 56(9225):233-41.

13. Lyi SM, Heller LI, Rutzke M, Welch RM, Kochian LV, Li L. Molecular and biochemical characterization of the selenocysteine Se-methyltransferase gene and Se-methylselenocysteine synthesis in broccoli. Plant Physiol. 2005; 138(1):409-20

14. Zhai L, Xu L, Wang Y, Zhu X, Feng H, Li C, Luo X, Everlyne MM, Liu L. Transcriptional identification and characterization of differentially expressed genes associated with embryogenesis in radish (Raphanus sativus L.). Sci Rep. 2016;6:21652.

15. Zhang N, Zhang HJ, Zhao B, Sun QQ, Cao YY, Li R, Wu XX, Weeda S, Li L, Ren S. The RNA-seq approach to discriminate gene expression profiles in response to melatonin on cucumber lateral root formation. J Pineal Re. 2014:56(1):39-50.

16. $\mathrm{Xu} \mathrm{H}$, Gao Y, Wang J. Transcriptomic analysis of rice (Oryza sativa) developing embryos using the RNA-Seq technique. PLoS One. 2012; 7(2):e30646

17. Nam J, dePamphilis CW, Ma H, Nei M. Antiquity and evolution of the MADS-box gene family controlling flower development in plants. Mol Biol Evol. 2003;20(9):1435-47.

18. Nakano T, Suzuki K, Fujimura T, Shinshi H. Genome-wide analysis of the ERF gene family in Arabidopsis and rice. Plant Physiol. 2006;140(2):411-32.
19. Eulgem T, Rushton PJ, Robatzek S, Somssich IE. The WRKY superfamily of plant transcription factors. Trends Plant Sci. 2000;5(5):199-206.

20. Yang J, Chen X, Zhu C, Peng X, He X, Fu J, Ouyang L, Bian J, Hu L, Sun X. RNA-seq reveals differentially expressed genes of rice (Oryza sativa) spikelet in response to temperature interacting with nitrogen at meiosis stage. BMC Genomics. 2015;16:959.

21. Agarwal P, Reddy MP, Chikara J. WRKY: its structure, evolutionary relationship, DNA-binding selectivity, role in stress tolerance and development of plants. Mol Biol Rep. 2011;38(6):3883-96.

22. Chen H, Lai Z, Shi J, Xiao Y, Chen Z, Xu X. Roles of arabidopsis WRKY18, WRKY40 and WRKY60 transcription factors in plant responses to abscisic acid and abiotic stress. BMC Plant Biol. 2010;10:281.

23. Ko SS, Li MJ, Sun-Ben Ku M, Ho YC, Lin YJ, Chuang MH, Hsing HX, Lien YC, Yang HT, Chang HC. The bHLH142 Transcription Factor Coordinates with TDR1 to Modulate the Expression of EAT1 and Regulate Pollen Development in Rice. Plant Cell. 2014;26(6):2486-504.

24. Inoue E. Transcriptome analyses give insights into selenium-stress responses and selenium tolerance mechanisms in Arabidopsis. Physiol Plant. 2010; 132(2):236-53.

25. Agarwal M, Hao YJ, Kapoor A, Dong CH, Fujii H, Zheng XW, Zhu JK. A R2R3 type MYB transcription factor is involved in the cold regulation of CBF genes and in acquired freezing tolerance. J Biol Chem. 2006;281 (49):37636-45.

26. Koes R, Verweij W, Quattrocchio F. Flavonoids: a colorful model for the regulation and evolution of biochemical pathways. Trends Plant Sci. 2005 10(5):236-42.

27. Hichri I, Heppel SC, Pillet J, Leon C, Czemmel S, Delrot S, Lauvergeat V, Bogs J. The basic helix-loop-helix transcription factor MYC1 is involved in the regulation of the flavonoid biosynthesis pathway in grapevine. Mol Plant. 2010;3(3):509-23.

28. Hoagland DR, Arnon DI. The water-culture method for growing plants without soil. Calif Agric Exp Stn Circ. 1950;347(5406):357-9.

29. Kallio MA, Tuimala JT, Hupponen T, Klemela P, Gentile M, Scheinin I, Koski M, Kaki J, Korpelainen El. Chipster: user-friendly analysis software for microarray and other high-throughput data. BMC Genomics. 2011;12:507.

30. Li B, Dewey CN. RSEM:Accurate transcript quantification from RNA-Seq data with or without a reference genome. BMC Bioinformatics. 2011;12:323.

31. Lu MW, Ngou FH, Chao YM, Lai YS, Chen NY, Lee FY, Chiou PP. Transcriptome characterization and gene expression of Epinephelus spp in endoplasmic reticulum stress-related pathway during betanodavirus infection in vitro. BMC Genomics. 2012;13(1):651-63.

32. Conesa A, Gotz S, Garcia-Gomez JM, Terol J, Talon M, Robles M. Blast2GO: a universal tool for annotation, visualization and analysis in functional genomics research. Bioinformatics. 2005;21(18):3674-6.

33. Kanehisa M, Araki M, Goto S, Hattori M, Hirakawa M, Itoh M, Katayama T, Kawashima S, Okuda S, Tokimatsu T. KEGG for linking genomes to life and the environment. Nucleic Acids Res. 2008;36:D480-4.

34. Deng YY, Li JQ, Wu SF, Zhu YP, Chen YW, He FC. Integrated nr database in protein annotation system and its localization. Comput Eng. 2006;32(5):71-2.

35. Kanehisa M, Goto S, Kawashima S, Okuno Y, Hattori M. The KEGG resource for deciphering the genome. Nucleic Acids Res. 2004;32(Database issue):277-80.

36. Altschul SF, Madden TL, Schaffer AA, Zhang JH, Zhang Z, Miller W, Lipman DJ. Gapped BLAST and PSI-BLAST: a new generation of protein database search programs. Nucleic Acids Res. 1997;25(17):3389-402.

37. Livak KJ, Schmittgen TD. Analysis of relative gene expression data using real-time quantitative PCR and the 2(-Delta Delta C(T)) method. Methods. 2001;25(4):402-8.

\section{Ready to submit your research? Choose BMC and benefit from:}

- fast, convenient online submission

- thorough peer review by experienced researchers in your field

- rapid publication on acceptance

- support for research data, including large and complex data types

- gold Open Access which fosters wider collaboration and increased citations

- maximum visibility for your research: over $100 \mathrm{M}$ website views per year

At $\mathrm{BMC}$, research is always in progress.

Learn more biomedcentral.com/submissions 